\title{
EURUSD INTRADAY PRICE REVERSAL
}

Marta Wiśniewska, Ph.D.

Gdansk School of Banking

Dolna Brama 8, 80-821 Gdańsk, Poland

e-mail:marta@witor.biz

Received 23 September 2014, Accepted 24 October 2014

\begin{abstract}
The study investigates the mean reversion in 1-minute EURUSD. Intraday patters in FX seem of particular interest as more and more trades in the FX market are automated high frequency trades (HFT). The study reveals that the mean reversion is present in the intraday EURUSD. ADF test rejects unit root. The average of the deviation of EURUSD from its (moving) mean is close to zero. Furthermore when short and long positions are simultaneously open, the average maximum return achieved through 24 hour period is similar, providing yet another evidence for mean reversion and lack of weak form of market efficiency.
\end{abstract}

Keywords: high frequency, intraday, price, EURUSD, reversal, mean, market efficiency.

JEL classification: G11, G14. 


\section{Introduction}

Foreign exchange (FX) market is the most liquid market in the world, with daily turnover reaching trillions of $\mathrm{USD}^{1}$. The FX market has undergone changes due to development in electronic trading 2 . It is estimated that the automated trades contribute to, depends on the currency, 40-60\% of the FX spot market transactions ${ }^{3}$. Moreover the share of high-frequency trading $(\mathrm{HFT})^{4}$ is constantly increasing. Yet HFT at the FX market is still not as popular as at the equity market ${ }^{5}$.

High share of automated trades and in particular the HFT means that the investment horizon of FX market participants has shortened rather drastically, to (often) several minutes ${ }^{6}$ Therefore, in order to understand the FX market dynamics, it seems appropriate to investigate the intraday data. The decisions of incising number of FX market participants are based on models employing intraday data, and computer algorithms dealing with intraday data.

There is rather extensive literature on factors affecting exchange rates, with monetary models being a constant source of discussion ever since 1970s ${ }^{7}$. Already in 1980s the power of the monetary models was challenged ${ }^{8}$. Although they might help to predict the long term behaviour of exchange rates, and the macroeconomics news announcements have also impact on the short term behaviour ${ }^{9}$, yet the classic linear models fail to explain the temporal fluctuations on FX market $^{10}$. It is believed that to capture those movements data of highest available frequency should be used ${ }^{11}$. Furthermore it has been shown that non-linear models can provide superior performance ${ }^{12}$.

This paper looks at high frequency (1-minute) EURUSD exchange rates ${ }^{13}$. In particular it tackles the problem whether mean reversion can be identified in this FX high frequency data. Intraday mean reversion can have powerful consequences for creating profitable (HFT) automated trades. At the same time mean revision is an argument against the weak form of market efficiency. Yet in the market with high share of HFT trades (that focus on exploring arbitrage possibilities and market imperfections) one would expect that market to be relatively efficient.

The paper is organized as follows: first the EURUSD intraday data (and its summary statistics) is briefly described. Next ADF tests are performed. Simultaneously the impact of moving averages on prices and the distribution of deviations from moving averages are outlined. Following that empirical investigation of two trading strategies (that utilize mean reversion) is undertaken. The paper outcomes are summarized in conclusions. 


\section{EURUSD intraday data}

Figure 1 presents the EURUSD 1-minute LastBid rates, while Table 1 displays their summary statistics. The mean exchange rate was 1.244315 USD per 1 EUR. Based on the diagram, it seems that in the long time horizon, the EURUSD is mean reverting around relatively constant mean of 1.244315. At the same time it appears that in the short time horizon, the EURUSD could be mean reverting, yet to a mean that is changing in time (i.e. EURUSD can be reverting to a moving average of some sort). The importance of moving average will be a subject of the next section.

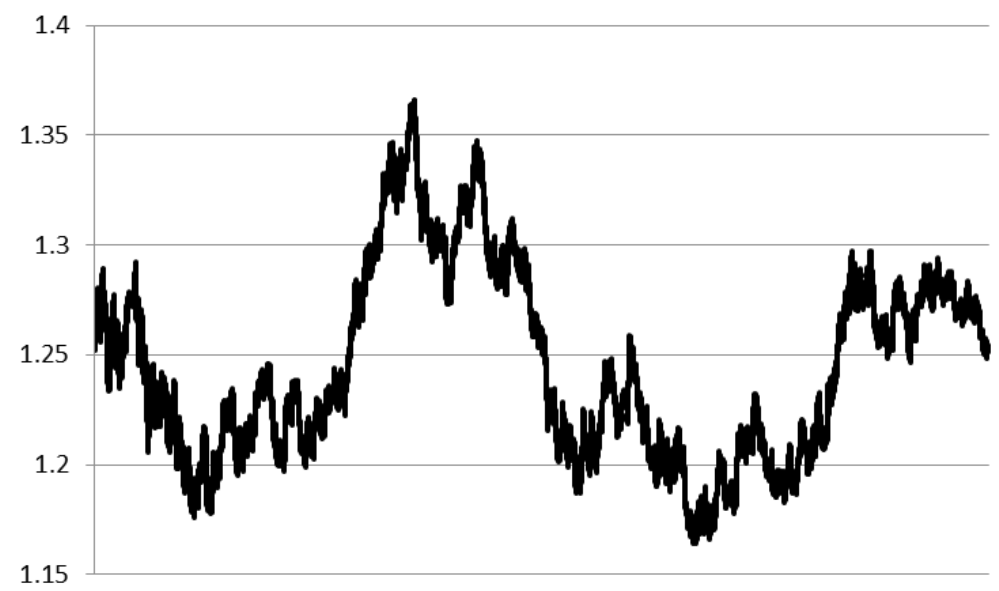

Fig. 1. EURUSD 1-minute LastBid, 2004-2006.

Source: own calculations.

Table 1. LastBid EURUSD, summary statistics

\begin{tabular}{|c|c|c|c|c|c|c|c|}
\hline Mean & \multicolumn{2}{|c|}{$[99.9 \%$ Conf. Interval $]$} & Std. Dev. & Min & Max & Skewness & Kurtosis \\
\hline 1.244315 & 1.244174 & 1.244456 & 0.043618 & 1.164 & 1.366 & 0.4668879 & 2.361226 \\
\hline
\end{tabular}

Source: own calculations.

High volatility of intraday exchange rate could be confirmed while comparing the spread between the maximum and minimum rate (i.e. $1.366-1.164=0.202)$ to the standard deviation. The standard deviation is equal to approximately 21.6 per cent of the spread, which is a relatively high number. 
The change of 0.202 (from 1.366) means a change of 14.7877 per cent, which in itself could be perceived as relatively small. Having said that, hence the FX trades are often conducted on leverage (for example $1: 25$ ), thus for many FX investors any change in the exchange rate will results in gains/losses magnified by the leverage ratio they use.

\section{Mean reversion}

Prior conducting any further research it is worthwhile noticing that the paper looks at the pattern in levels (i.e. prices) as opposed to returns. This is not common for financial markets but it is popular in commodity market ${ }^{14}$. This approach was selected due to the fact that in previous section a mean reversion in prices was identified.

According to the results of ADF test presented in Table 2, the null hypothesis of unit root can be rejected for ADF test with a drift. Thus EURUSD was created by stationary process with a drift. This in itself implies mean reversion (to a mean that is changing in time), and confirms earlier observation that in sort horizon EURUSD is mean reverting to a mean that is changing in time. Reminder of this section will investigate the importance of moving average and the distribution of deviations from moving average.

Table 2. LastBid EURUSD, ADF test

\begin{tabular}{|l|l|c|c|c|}
\cline { 3 - 5 } \multicolumn{2}{c|}{} & $\operatorname{lags}(10)$ & $\operatorname{lags}(20)$ & $\operatorname{lags}(60)$ \\
\hline \multirow{2}{*}{ p-value } & with drift & 0.0091 & 0.0094 & 0.0094 \\
\cline { 2 - 5 } & without drift & 0.1525 & 0.1566 & 0.1559 \\
\hline
\end{tabular}

Source: own calculations.

Table 3 displays the results of the regression with: (1) moving average of (x) LastBid rates as an explanatory variable ${ }^{15},(2)$ as (1) but additionally include: D1 dummy variable that is equal to 1 if the LastBid has increased at $t=0$, or $D 2$ dummy variable that is equal to 1 if the LastBid increased at $t=-1$ :

$$
\operatorname{LastBid}_{t}=\alpha_{1}+\alpha_{2} \operatorname{malBid}(x)_{t}+\alpha_{3} D 1_{t}+\alpha_{4} D 2_{t}+\varepsilon_{t}
$$

The moving average as an explanatory variable contributes to over $95 \%$ in the variation of LastBid. Changes in the moving average result in changes in LastBid of similar magnitude. Furthermore, partial autocorrelation function for LastBid is finished and it is disappearing (i.e. it is equal to zero) after 2 lags, suggesting $\mathrm{AR}(2)$ process. 
Table 3. Moving average regression

\begin{tabular}{|l|c|c|c|c|}
\hline \multicolumn{1}{|c|}{ Explanatory variable } & $\alpha_{2}$ & Adj R-square & D1 & D2 \\
\hline maLBid60 & $0.9998813^{* * *}$ & 0.9996 & 0.0002290 & 0.0001542 \\
\hline maLBid120 & $0.9997656^{* * *}$ & 0.9992 & 0.0002195 & 0.0001466 \\
\hline maLBid180 & $0.9996456 * * *$ & 0.9989 & 0.0002130 & 0.0001408 \\
\hline maLBid240 & $0.9995281 * * *$ & 0.9985 & 0.0002091 & 0.0001372 \\
\hline maLBid300 & $0.9994118^{* * *}$ & 0.9981 & 0.0002060 & 0.0001343 \\
\hline maLBid360 & $0.9992964 * * *$ & 0.9977 & 0.0002034 & 0.0001318 \\
\hline maLBid720 & $0.9986504 * * *$ & 0.9955 & 0.0001962 & 0.0001249 \\
\hline maLBid24h & $0.9973856 * * *$ & 0.9909 & 0.0001920 & 0.0001208 \\
\hline maLBid48h & $0.9954024 * * *$ & 0.9821 & 0.0001838 & 0.0001127 \\
\hline maLBid72h & $0.9926887 * * *$ & 0.9740 & 0.0001761 & 0.0001050 \\
\hline maLBid4d & $0.9910969 * * *$ & 0.9663 & 0.0001875 & 0.0001164 \\
\hline maLBid5d & $0.9896982 * * *$ & 0.9591 & 0.0001908 & 0.0001201 \\
\hline
\end{tabular}

*** Significant at $99 \%$ significance level.

Source: own calculations.

While investigating the mean reversion it could prove useful to look at the distribution of the deviations from the moving average (for different time horizons $x$ ). Table 4 shows that both the mean and the median of deviations of various $x$ are very close to zero. The kurtosis of the distribution for low $x$ is relatively high, implying fat tails. The kurtosis is decreasing with $x$, thus the higher the $x$ the less frequent are extreme (much varying from moving average) prices. This yet again confirms mean reversion in high frequency data.

Table 4. LastBid deviations from the (x) moving average

\begin{tabular}{|c|c|c|c|c|c|c|c|c|c|}
\hline \multirow{3}{*}{$\mathrm{d} 60$} & \multicolumn{3}{|c|}{ Percentile } & \multicolumn{3}{|c|}{ Mean } & \multirow{3}{*}{\begin{tabular}{l|} 
Std Dev \\
0.0008526
\end{tabular}} & \multirow{3}{*}{$\begin{array}{l}\text { Skewness } \\
0.1326834 \\
\end{array}$} & \multirow{3}{*}{$\begin{array}{c}\text { Kurtosis } \\
19.40152 \\
\end{array}$} \\
\hline & \multirow{2}{*}{\begin{tabular}{|c|}
$1 \%$ \\
-0.0024250 \\
\end{tabular}} & \multirow{2}{*}{\begin{tabular}{|c|}
$50 \%$ \\
$-1.09 \mathrm{E}-08$ \\
\end{tabular}} & \multirow{2}{*}{$\begin{array}{c}99 \% \\
0.0024650\end{array}$} & & \multicolumn{2}{|c|}{$99 \%$ Conf. Interval } & & & \\
\hline & & & & $-5.41 \mathrm{E}-06$ & $-7.83 \mathrm{E}-06$ & $-2.99 \mathrm{E}-06$ & & & \\
\hline $\mathrm{d} 120$ & -0.0034616 & $-3.81 \mathrm{E}-08$ & 0.0035232 & $-1.05 \mathrm{E}-05$ & $-1.39 \mathrm{E}-05$ & $-7.16 \mathrm{E}-06$ & 0.0011979 & 0.1035210 & 13.78254 \\
\hline $\mathrm{d} 180$ & -0.0042705 & $-3.81 \mathrm{E}-08$ & 0.0043427 & $-1.57 \mathrm{E}-05$ & $-1.98 \mathrm{E}-05$ & $-1.16 \mathrm{E}-05$ & 0.0014635 & 0.1345139 & 11.26028 \\
\hline $\mathrm{d} 360$ & -0.0059374 & $-7.81 \mathrm{E}-06$ & 0.0060570 & $-3.11 \mathrm{E}-05$ & $-3.69 \mathrm{E}-05$ & $-3.69 \mathrm{E}-05$ & -0.0000253 & 0.0934773 & 8.15897 \\
\hline $\mathrm{d} 720$ & -0.0079390 & -0.0000155 & 0.0082538 & $-5.79 \mathrm{E}-05$ & $-6.60 \mathrm{E}-05$ & $-6.60 \mathrm{E}-05$ & -0.0000497 & 0.0629643 & 5.771617 \\
\hline $\mathrm{d} 24 \mathrm{~h}$ & -0.0105027 & 0.0000162 & 0.0107106 & $-8.99 \mathrm{E}-05$ & $-1.02 \mathrm{E}-04$ & $-7.82 \mathrm{E}-05$ & 0.0041662 & -0.0183756 & 4.161308 \\
\hline $\mathrm{d} 48 \mathrm{~h}$ & -0.0146764 & -0.0000244 & 0.0138367 & -0.0001507 & -0.0001672 & -0.0001342 & 0.0058508 & -0.0670965 & 3.38424 \\
\hline $\mathrm{d} 72 \mathrm{~h}$ & -0.0176475 & -0.0001505 & 0.0157654 & -0.0002241 & -0.0002441 & -0.0002041 & 0.0070489 & -0.1210407 & 3.157879 \\
\hline $\mathrm{d} 4 \mathrm{~d}$ & -0.0200260 & -0.0002817 & 0.0173793 & -0.000307 & -0.0003298 & -0.0002842 & 0.0080355 & -0.1214906 & 2.980011 \\
\hline $\mathrm{d} 5 \mathrm{~d}$ & -0.0220042 & -0.0004817 & 0.0184969 & -0.0003637 & -0.0003889 & -0.0003889 & -0.0003385 & -0.1372214 & 2.916339 \\
\hline
\end{tabular}

$\mathrm{d} 60$ stands for the difference between LastBid and maLBid60.

Source: own calculations. 
Following section will provide an additional evidence for mean reversion properties of the 1-minute LastBid series. It investigates trading strategy where simultaneously short and long positions are opened. The positions are to be held for maximum 24 hours, and the possible returns resulting from those positions are compared. If both positions yield similar returns this surely must be due to intraday mean reversion in EURUSD.

\section{Trading strategy vs. mean reversion}

Assume there are two possible trading strategies: (1) going long strategy (BS) where EURUSD is purchased initially (at ask rate A at time $t=1$ ) and then sold (at bid rate $b_{t}$ at some future time $t=n$, where $n=2, \ldots, 1,440$ ), and (2) short sell strategy (SS) where EURUSD is sold (at a bid rate $B$ at time $t=1$ ) and then repurchased back (at ask rate $\mathrm{a}_{\mathrm{t}}$ at time $t=n$, where $n=2, \ldots, 1,440)^{16}$. For each trading day and each strategy there are 1,439 possible returns calculated as:

$$
R_{B S}(t)=b_{t}-A
$$

and

$$
R_{S S}(t)=B-\alpha_{t}
$$

where $R_{B S}(t)$ is the return coming from (1) strategy achieved ${ }^{17}$ at time $t=n$ (where $n=2, \ldots, 1,440$ ) and $R_{S S}(t)$ is the return coming from the (2) strategy achieved at time $t=n$ (where $n=2, \ldots, 1,440$ ).

Tables 5 and 6 outlines the summary statistics of maximum returns possible to achieve by following BS (Table 5) or SS (Table 6) strategies for 24 hours. The study allows for changing the time when the position was initially opened. There are 24 possible position opening time (o). They were selected as 1st minute of each hour. Executing both trading strategies allow for positive returns. The average maximum return for BS strategy is equal to 0.005525 (with $99 \%$ confidence interval of $(0.005501 ; 0.005548)$. This is lower than the average maximum return for SS strategy, which is equal to 0.006046 , with the $99 \%$ confidence interval being $(0.006023$; 0.006069). The average variation between the maximum returns of both strategies is equal to approximately 9 per cent of the return. Similar results coming from following BS and SS strategies seem only possible in case of mean reversion. Thus the above results confirm once again the intraday mean reversion established in the previous section. 
Table 5. Descriptive statistics for maximum returns achieved through 24 hours period due $\mathrm{t}$ o following BS strategy dependent on time (o) the position was initially opened

\begin{tabular}{|c|c|c|c|c|c|c|}
\hline o & Mean & \multicolumn{2}{|c|}{$99 \%$ Conf. Interval } & Std. Dev. & Min & Max \\
\hline 0 & 0.00486 & 0.004837 & 0.004884 & 0.004280 & -0.0005 & 0.0203 \\
\hline 1 & 0.006248 & 0.006222 & 0.006275 & 0.004795 & -0.0003 & 0.0258 \\
\hline 2 & 0.006276 & 0.006250 & 0.006302 & 0.004698 & -0.0004 & 0.0261 \\
\hline 3 & 0.006174 & 0.006149 & 0.006200 & 0.004612 & -0.0002 & 0.0249 \\
\hline 4 & 0.006065 & 0.006040 & 0.006090 & 0.004522 & -0.0004 & 0.0228 \\
\hline 5 & 0.005942 & 0.005917 & 0.005966 & 0.004446 & -0.0004 & 0.0224 \\
\hline 6 & 0.005861 & 0.005836 & 0.005886 & 0.004530 & -0.0002 & 0.0217 \\
\hline 7 & 0.006013 & 0.005988 & 0.006038 & 0.004551 & -0.0003 & 0.0221 \\
\hline 8 & 0.006039 & 0.006015 & 0.006063 & 0.004365 & -0.0003 & 0.0207 \\
\hline 9 & 0.005909 & 0.005884 & 0.005933 & 0.004452 & -0.0004 & 0.0219 \\
\hline 10 & 0.005720 & 0.005695 & 0.005744 & 0.004429 & -0.0003 & 0.0209 \\
\hline 11 & 0.005754 & 0.005730 & 0.005779 & 0.004418 & -0.0003 & 0.0222 \\
\hline 12 & 0.005932 & 0.005908 & 0.005955 & 0.004301 & -0.0003 & 0.0224 \\
\hline 13 & 0.005963 & 0.005938 & 0.005987 & 0.004385 & -0.0004 & 0.0223 \\
\hline 14 & 0.005598 & 0.005575 & 0.005620 & 0.004094 & -0.0004 & 0.0219 \\
\hline 15 & 0.005475 & 0.005453 & 0.005497 & 0.004042 & -0.0004 & 0.0215 \\
\hline 16 & 0.005351 & 0.005329 & 0.005373 & 0.003937 & -0.0005 & 0.0236 \\
\hline 17 & 0.005080 & 0.005058 & 0.005102 & 0.003938 & -0.0003 & 0.0195 \\
\hline 18 & 0.004986 & 0.004964 & 0.005008 & 0.003999 & -0.0002 & 0.0198 \\
\hline 19 & 0.004763 & 0.004740 & 0.004785 & 0.004050 & -0.0003 & 0.0200 \\
\hline 20 & 0.004692 & 0.004669 & 0.004716 & 0.004211 & -0.0004 & 0.0247 \\
\hline 21 & 0.004607 & 0.004583 & 0.004630 & 0.004266 & -0.0003 & 0.0238 \\
\hline 22 & 0.004631 & 0.004607 & 0.004655 & 0.004321 & -0.0004 & 0.0227 \\
\hline 23 & 0.004650 & 0.004626 & 0.004674 & 0.004297 & -0.0005 & 0.0209 \\
\hline Average & 0.055250 & 0.005501 & 0.005548 & & & \\
\hline Min & 0.004607 & 0.004583 & 0.004630 & & & \\
\hline Max & 0.006276 & 0.006250 & 0.006302 & & & \\
\hline
\end{tabular}

Source: own calculations.

Table 6. Descriptive statistics for maximum returns achieved through 24 hours period due to following SS strategy dependent on time (o) the position was initially opened

\begin{tabular}{|l|c|c|c|c|c|c|}
\hline \multirow{2}{*}{\begin{tabular}{c} 
o \\
\hline
\end{tabular}} & Mean & \multicolumn{2}{|c|}{$99 \%$ Conf. Interval } & Std. Dev. & Min & Max \\
\hline 0 & 2 & 3 & 4 & 5 & 6 & 7 \\
\hline 1 & 0.004990 & 0.004967 & 0.005013 & 0.004158 & -0.0005 & 0.0214 \\
\hline 2 & 0.006423 & 0.006400 & 0.006447 & 0.004297 & -0.0004 & 0.0206 \\
\hline 3 & 0.006365 & 0.006341 & 0.006388 & 0.004282 & -0.0001 & 0.0213 \\
\hline 4 & 0.006536 & 0.006512 & 0.006559 & 0.004243 & -0.0003 & 0.0229 \\
\hline 5 & 0.006580 & 0.006557 & 0.006603 & 0.004238 & -0.0004 & 0.0230 \\
\hline
\end{tabular}




\begin{tabular}{|c|c|c|c|c|c|c|}
\hline 1 & 2 & 3 & 4 & 5 & 6 & 7 \\
\hline 6 & 0.006654 & 0.0066310 & 0.0066770 & 0.004171 & -0.0002 & 0.0237 \\
\hline 7 & 0.006568 & 0.0065460 & 0.0065900 & 0.003992 & -0.0003 & 0.0207 \\
\hline 8 & 0.006888 & 0.0068650 & 0.0069110 & 0.004086 & -0.0003 & 0.0225 \\
\hline 9 & 0.006862 & 0.0068390 & 0.0068850 & 0.004133 & -0.0004 & 0.0208 \\
\hline 10 & 0.006827 & 0.0068050 & 0.0068490 & 0.004007 & -0.0002 & 0.0201 \\
\hline 11 & 0.006652 & 0.0066290 & 0.0066760 & 0.004268 & -0.0001 & 0.0208 \\
\hline 12 & 0.006329 & 0.0063060 & 0.0063530 & 0.004257 & -0.0003 & 0.0197 \\
\hline 13 & 0.006013 & 0.0059890 & 0.0060360 & 0.004272 & -0.0006 & 0.0199 \\
\hline 14 & 0.005885 & 0.0058620 & 0.0059080 & 0.004189 & -0.0003 & 0.0193 \\
\hline 15 & 0.005575 & 0.0055530 & 0.0055970 & 0.004050 & -0.0002 & 0.0208 \\
\hline 16 & 0.005498 & 0.0054760 & 0.0055200 & 0.003969 & -0.0001 & 0.0210 \\
\hline 17 & 0.005374 & 0.0053520 & 0.0053960 & 0.003962 & -0.0001 & 0.0215 \\
\hline 18 & 0.005402 & 0.0053800 & 0.0054240 & 0.003955 & -0.0002 & 0.0227 \\
\hline 19 & 0.005431 & 0.0054090 & 0.0054530 & 0.003972 & -0.0003 & 0.0229 \\
\hline 20 & 0.005401 & 0.0053790 & 0.0054220 & 0.003893 & -0.0001 & 0.0223 \\
\hline 21 & 0.005442 & 0.0054200 & 0.0054640 & 0.004008 & -0.0005 & 0.0214 \\
\hline 22 & 0.005454 & 0.0054310 & 0.0054770 & 0.004124 & -0.0003 & 0.0209 \\
\hline 23 & 0.005293 & 0.0052700 & 0.0053160 & 0.004145 & -0.0003 & 0.0210 \\
\hline Average & 0.006046 & 0.006023 & 0.006069 & & & \\
\hline Min & 0.004990 & 0.004967 & 0.005013 & & & \\
\hline Max & 0.006888 & 0.006865 & 0.006911 & & & \\
\hline
\end{tabular}

Source: own calculations.

There are some limitations of the above analysis. The study assumes 24 hours as the maximum period of holding an open position. As has been indicated previously, HFT can focus on much shorter horizon, therefore investigation of performance of BS and SS strategies for shorter horizon would seem justified. At the same time it is worth noting that the minimum values of the maximum returns can be far below the mean, and can reach negative values (Table 5). Such negative observations are rare, nevertheless they do exist. It seems interesting to investigate what is the time needed for mean reversion to occur in such circumstances? Further studies could address this question.

\section{Conclusions}

The study tackles the problem of mean reversion in high frequency data. In particular it investigates the 1-minute EURUSD exchange rate. Intraday patters in FX seem of particular interest as more and more trades in the FX market are automated HFT.

The ADF test revealed that the EURUSD is created by a stationary process with a drift. At the same time moving averages are important when explaining EURUSD. Furthermore the distribution of the EURUSD deviations from the mean suggest the average deviation is very 
close to zero. On basis of all this observations it can be confirmed that the EURUSD experience a mean reversion. Further confirmation steams from opening simultaneously short and long position in EURUSD. It has been revealed that, on average, both positions can provide similar positive returns. That would not be a case if EURUSD was not mean reverting.

The mean reversion can be used for creating trading strategies that would provide abnormal returns. Therefore discovering this pattern provides and evidence for violation of weak form of market efficiency. It seems that EURUSD is not efficient in intraday horizon. Further studies could explore the benefits of strategies resulting from this inefficiency.

The study features several limitations, some outlined in the section above. It would seem further investigation of the topic is fully justify. In particular it would be interesting to know how robust in time the results are. Thus if longer and/or more recent dataset was used for the analysis would the results be intact? Moreover would there be an impact if the provider of the data (the EURUSD quotes) was changed? As FX is an OTC market that can have an implication on the market efficiency as well.

\section{Notes}

${ }^{1}$ The average daily turnover for April 2010 was 3.98 USD (BIS, 2011).

${ }^{2}$ Electronic trading can be divided into manual and automated. In manual trade instructions are executed by humans on the electronic trading platform, whereas in automated they are executed by computer algorithms (BIS, 2011).

${ }^{3}$ Where spot market is making up for 39\% of FX trades in 2010 (BIS, 2011).

${ }^{4}$ HFT are often perceived as automated trading, where decision-making is taken over by computer algorithm. According to Lee (2010) HFT is $25 \%$ of all FX activity.

${ }^{5}$ HFT represents $56 \%$ of the US equity market, $38 \%$ of European markets and in the range of $10-30 \%$ of Asia-Pacific markets (IOSCO, 2011).

${ }^{6}$ This seems particularly true for those participants treating FX market as a market of an investment asset.

${ }^{7}$ Dornbusch (1976); Frenkel (1976); Mussa (1976); Bilson (1978).

${ }^{8}$ Messe, Rogoff (1983).

${ }^{9}$ Andersen et al. (2003), (2007); Faust et al. (2007).

10 Taylor, Taylor (2004).

11 Dempster et al. (2001).

12 Boero, Marrocu (2002); Kamruzzaman, Sarker (2004).

${ }^{13}$ Last Bid and Last Ask rates for 1-minute horizon were obtained from Bank of America. There are 1,440 observations in each trading day. The dataset cover years 2004-2006.

${ }^{14}$ For oil market see Cartea, Figueroa (2005) or Opalski et al. (2011).

15 MaLBid60 stands for moving average of LastBid for $t$ from -60 to -1 .

${ }^{16}$ Both ask and bid rates are the Last rates for the 1-minute interval provided by Bank of America. Due to data availability and to facilitate the calculation the study period covers trading days in 2005 only.

17 The position is being closed out at time $t=n$. 


\section{References}

Andersen, T.G., Bollerslev, T., Diebold, F.X. \& Vega, C. (2003). Micro effects of macro announcements: real time price discovery in foreign exchange. American Economic Review, 93 (1), 38-62.

Andersen, T.G., Bollerslev, T., Diebold, F.X. \& Vega, C. (2007). Real-time price discovery in global stock, bond and foreign exchange markets. Journal of International Economics, $73,251-277$.

BIS (2011). Bank for International Settlements. High-frequency trading in the foreign exchange market, BIS, September.

Boero, G. \& Marrocu, E. (2002). The performance of non linear exchange rate models: A forecasting comparison. Journal of Forecasting, 21, 513-542.

Bilson, J.F.O. (1978). Rational Expectations and the Exchange Rate. In: The Economics of Exchange Rates: Selected Studies, eds. J.A. Frenkel, H.G. Johnson. Reading, MA: AddisonWesley Press.

Cartea, A. \& Figueroa, M.G. (2005). Pricing in Electricity Markets: a mean reverting jump diffusion model with seasonality. Applied Mathematical Finance, 12, 313-335.

Dempster, M.A.H., Payne, T.W., Romahi, Y. \& Thompson, G.W.P. (2001). Computational learning techniques for intraday FX trading using popular technical indicators. IEEE Transactions on Neural Networks, 12 (4), 744-754.

Dornbusch, R. (1976). Expectations and Exchange Rate Dynamics. Journal of Political Economy, 84 (6), 1161-1176.

Faust, J., Rogers, J.H., Wang, S.Y.B. \& Wright, J.H. (2007). The high-frequency response of exchange rates and interest rates to macroeconomic announcements. Journal of Monetary Economics, 54, 1051-1068.

Frenkel, J.A. (1976). A Monetary Approach to the Exchange Rate: Doctrinal Aspects and Empirical Evidence. Scandinavian Journal of Economics, 78 (2), 200-224.

Kamruzzaman, J. \& Sarker, R.A. (2004). ANN based forecasting of foreign currency exchange rates. Neural Information Processing - Letters and Reviews, 3 (2), 49-58.

IOSCO (2011). International Organization of Securities Commissions, Regulatory issues raised by the impact of technological changes on market integrity and efficiency. Consultation report by the Technical Committee of the IOSCO, CR02/11, July 2011.

Lee, S. (2010). High-frequency trading in FX: open for business. Aite Group Impact Note, April.

Meese, R.A. \& Rogoff, K.S. (1983). Empirical exchange rate models of the seventies. Do they fit out of sample? Journal of International Economics, 14 (1-2), 3-24. 
Mussa, M. (1976). The Exchange Rate, the Balance of Payments and Monetary and Fiscal Policy under a Regime of Controlled Floating. Scandinavian Journal of Economics, 78 (2), 229-248.

Opalski, K., Kacprzak, K., Maciejczyk, K. \& Pawlowski, M. (2011). Modelowanie polskiego rynku energii elektrycznej. Uniwersytet Warszawski, Matematyka Stosowana, T. 1/54.

Taylor, A.M. \& Taylor, M.P. (2004). The purchasing power parity debate. The Journal of Economic Perspectives, 18, 135-158. 